\title{
Demodulation of carrier fringe patterns by the use of non-recursive digital phase locked loop
}

\author{
M. Servin ${ }^{\mathrm{a}, *}$, J.A. Quiroga ${ }^{\mathrm{b}}$, F.J. Cuevas ${ }^{\mathrm{a}}$ \\ a Centro de Investigaciones en Óptica A.C., Apartado Postal 1-948, 37150 León, Guanajuato, Mexico \\ ${ }^{\mathrm{b}}$ Departamento de Óptica, Universidad Complutense de Madrid, Ciudad Universitaria S/N, 28040 Madrid, Spain
}

Received 15 August 2001; received in revised form 1 October 2001; accepted 3 October 2001

\begin{abstract}
First- and second-order recursive Digital Phase Locked Loops (DPLLs) have been used recently in fringe data processing because it is the fastest way to obtain the unwrapped phase of a carrier frequency fringe pattern due to its minimal computational overhead. Nevertheless these simple DPLLs cannot cope with fringes having high noise and very wide band phase modulation. In this work we present a highly improved DPLL. The system presented is a nonrecursive DPLL which is far more robust than previously presented recursive DPLL. The advantage of this newer technique with respect to recursive DPLL is its higher gain in the signal to noise ratio on the detected phase and higher stability. Unfortunately this is obtained at a higher computational cost. (C) 2001 Published by Elsevier Science B.V.
\end{abstract}

PACS: $42.30 ; 42.30 . \mathrm{R} ; 42.87$

Keywords: Image processing in optics; Phase retrieval; Optical testing

\section{Introduction}

Recently a recursive second-order Digital Phase Locked Loop (DPLL) was used to successfully demodulate projection fringes at video rates by Gdeisat et al. [1]. This is possible because of the little computation overhead that these systems demand to demodulate carrier frequency fringes.

The DPLL applied to the demodulation of carrier frequency fringe patterns was introduced in 1993 by Servin and Rodriguez-Vera [2]. The sim-

\footnotetext{
${ }^{*}$ Corresponding author. Fax: +52-47-175000.

E-mail address: mservin@cio.mx (M. Servin).
}

plest possible DPLL is the first-order DPLL (Fig. 1). The main advantage of the DPLL approach is its very fast phase estimation that can be easily implemented to analyze two-dimensional fringe patterns at video rates using pipeline computing devices. Additionally the process of demodulation and unwrapping is made simultaneously, so no further unwrapping process is required. Recently Gdeisat et al. [1] have reported a second-order DPLL which is more robust and stable than the first-order DPLL but nevertheless it is still a weak system that cannot deal with high noise and/or wide band signals.

The first- and second-order DPLLs applied to interferometry were taken almost without change 


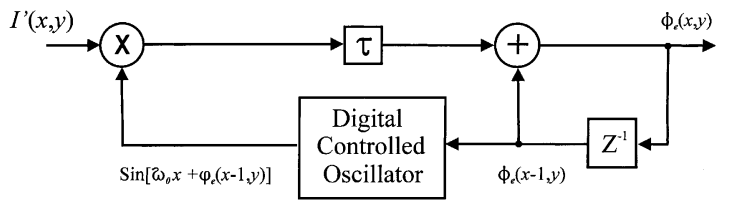

Fig. 1. Block diagram of a first-order digital phase locked loop (DPLL). $\phi_{\mathrm{e}}(x, y)$ is the estimated phase, the block containing $Z^{-1}$ represents a one pixel delay, and finally $\tau$ is the closed loop gain of the DPLL.

from the recursive phase estimators used in Communication engineering. In electrical communications recursive estimator is a natural choice given that the data and the estimated phase signals are temporal. The only thing that was changed to adapt the temporal DPLL to a spatial DPLL was that the temporal signal was replaced by a onedimensional spatial signal. This temporality issue is limited because information is available just from past and present. In other words, the temporal DPLL depends only on current data and previously estimated data to estimate the current phase. For example, the first-order temporal DPLL uses only the previously estimated data and current fringe pixel to estimate the current phase; a second-order DPLL depends on two previously estimated phases and the current fringe pixel. Nevertheless in fringe pattern analysis we are not dealing with temporal signals but with spatial twodimensional signals. This fact may be used to our advantage to propose a more robust two-dimensional DPLL. So in this work we propose a nonrecursive two-dimensional DPLL (NRDPLL) that outperforms the previously reported DPLL. The NRDPLL proposed herein takes full advantage of the fact that the signal being processed is twodimensional.

\section{Carrier frequency interferograms}

Carrier frequency interferograms are obtained by adding a large tilt to the reference beam in an interferometer to obtain almost straight fringes phase modulated by the wavefront under test. A carrier frequency interferogram may be represented by
$I(x, y)=a(x, y)+b(x, y) \cos \left(\omega_{0} x+\phi(x, y)\right)$,

where $\omega_{0}$ is the carrier frequency introduced by the tilted reference. $a(x, y)$ and $b(x, y)$ are the low frequency functions that represent the background illumination variations and local contrast, respectively. Finally $\phi(x, y)$ is the phase field that one tries to recover. The carrier frequency must be greater than the maximum frequency content of the modulating phase in the direction of the introduced carrier, that is,

$\omega_{0}>\left.\frac{\partial \phi(x, y)}{\partial x}\right|_{\operatorname{Max}}$,

where Max is the maximum value that the modulating phase derivative takes within the area of interest.

To simplify our analysis for the rest of the paper let us assume that the term $a(x, y)$ has been filtered out by a proper digital high pass filter, and that the contrast term $b(x, y)$ has been normalized. After eliminating the low frequency term $a(x, y)$ and normalizing the amplitude function $b(x, y)$ one may approximate the carrier frequency fringe pattern as

$I^{\prime}(x, y) \approx \cos \left(\omega_{0} x+\phi(x, y)\right)$.

There exist several ways of demodulating carrier frequency interferograms. Among the best known are the Fourier technique [3], and the Synchronous technique [4]. These two well-known techniques use linear quadrature filters to detect the interesting phase. These systems also give the demodulated phase wrapped due to the arc tangent function required. As a consequence a further unwrapping step is required in the phase estimation process to obtain the desired continuous phase.

\section{Review of recursive DPLL}

For the reader's convenience and to better understand the advantages as well as the consequences of the proposed NRDPLL we briefly review the theory behind the recursive DPLL as applied to demodulate carrier frequency interferograms. The basic principle behind the DPLL is to track the phase changes of the input signal by 
varying the phase of a reference signal. The reference signal must have the same carrier frequency $\omega_{0}$ as the interferogram under analysis in such a way that only the phase changes of the carrier are followed.

From the block diagram shown in Fig. 1 we may write the digital non-linear equation for a first-order PLL as

$$
\begin{aligned}
\phi_{\mathrm{e}}(x, y)= & \phi_{\mathrm{e}}(x-1, y)+\mu I^{\prime}(x, y) \\
& \times \sin \left[\omega_{0} x+\phi_{\mathrm{e}}(x-1, y)\right],
\end{aligned}
$$

using as initial condition,

$\phi_{\mathrm{e}}\left(x_{0}, y_{0}\right)=0$.

As we can see from this equation the currently estimated phase $\phi_{\mathrm{e}}(x, y)$ depends on the previously estimated phase $\phi_{\mathrm{e}}(x-1, y)$ as well as on a product from the current data $I^{\prime}(x, y)$ and the reference signal $\sin \left[\omega_{0} x+\phi_{\mathrm{e}}(x-1, y)\right]$. The parameter $\mu$ is related with the bandwidth of the first-order recursive filter and it is typically fixed to a value less than 1 to ensure stability. A small value of it will give a system high inertia (a narrow bandwidth) so a high signal to noise ratio in the estimated phase is obtained. But on the other hand choosing a higher value of $\mu$, rapid spatial phase changes may be followed but also the DPLL may leak some carrier signal and too much noise. So there is a compromise between noise rejection and wide band phase tracking.

The previously estimated phase $\phi_{\mathrm{e}}(x-1, y)$ can be viewed as a first-order predictor (or regularizer) of the current phase estimate. The second term contains the data $I^{\prime}(x, y)$ which are multiplied by the reference signal that tracks the modulating phase. Computationally speaking this DPLL is an extremely efficient system given that only a sum and a multiplication are needed to estimate the current phase.

Given that the DPLL is a one-dimensional sequential recursive phase estimator, a two-dimensional scanning sequence is required. Each line is scanned twice because at the beginning the recursive system will have a transient response so its estimated phase may not be valid, so each line must be scanned forward and backward. The backward estimated phase is the one that is kept as valid. This scanning procedure nevertheless decreases by half the DPLL computational efficiency. This double scanning annoyance is also eliminated by the non-recursive DPLL proposed in this work.

\section{Analysis of recursive DPLL}

In this section we review the basic concepts behind the functioning of the DPLL. The DPLL may be analyzed if one substitutes the high pass filtered irradiance given in Eq. (3) into Eq. (4) obtaining

$$
\begin{aligned}
\phi_{\mathrm{e}}(x, y)= & \phi_{\mathrm{e}}(x-1, y)+\mu \cos \left[\omega_{0} x+\phi(x, y)\right] \\
& \times \sin \left[\omega_{0} x+\phi_{\mathrm{e}}(x-1, y)\right],
\end{aligned}
$$

and this in turn may be expanded to obtain

$$
\begin{aligned}
\phi_{\mathrm{e}}(x, y)= & \phi_{\mathrm{e}}(x-1, y) \\
& +\frac{\mu}{2}\left\{\sin \left[\phi(x, y)-\phi_{\mathrm{e}}(x-1, y)\right]\right. \\
& \left.+\sin \left[2 \omega_{0} x+\phi(x, y)+\phi_{\mathrm{e}}(x-1, y)\right]\right\} .
\end{aligned}
$$

The second term in this equation is a low frequency term which is not affected by the recursive low pass first-order filter. The third term is a high frequency signal having a carrier frequency twice as high as the original fringes, so this term is highly attenuated. So Eq. (6) reduces to

$$
\begin{aligned}
\phi_{\mathrm{e}}(x, y)= & \phi_{\mathrm{e}}(x-1, y) \\
& +\frac{\mu}{2} \sin \left[\phi(x, y)-\phi_{\mathrm{e}}(x-1, y)\right] .
\end{aligned}
$$

A better insight into the inner working of the DPLL may be obtained from the last equation which gives the current phase estimate as a sum of two terms: The first one may be seen as a first-order linear predictor (or regularizer) which forces the current estimated phase to be close to the previously estimated one in the Euclidean space. The second term stands for the error (measured in the wrapped phase space) between the actual phase and the previously demodulated one. 
The DPLL technique has been extended by Kozlowski and Serra [5] to cope with higher phase variations and recently by Gdeisat et al. [1] by considering a second-order DPLL with improved performance over the first-order DPLL [2]. The second-order DPLL as reported in [1] may be stated as follows:

$$
\begin{aligned}
\phi_{\mathrm{e}}(x, y)= & \phi_{\mathrm{e}}(x-1, y) \\
& +\mu_{1} I^{\prime}(x, y) \sin \left[\omega_{0} x+\phi_{\mathrm{e}}(x-1, y)\right] \\
& +s(x, y), \\
s(x, y)=s & (x-1, y) \\
& +\mu_{2} I^{\prime}(x, y) \sin \left[\omega_{0} x+\phi_{\mathrm{e}}(x-1, y)\right],
\end{aligned}
$$

where the two constants $\mu_{1}$ and $\mu_{2}$ control the bandwidth of the estimated phase $\phi_{\mathrm{e}}(x, y)$. One important difference between the first-order DPLL and the second-order one is the order of the recursive low pass filter. In the first-order DPLL a first-order recursive digital filter is used to filter out the spurious double frequency signal. Using a second-order low pass recursive filter will have in general a sharper cutoff so the noise and second harmonics rejection in the estimated phase is superior to that of a first-order DPLL. Because the second-order filter requires two successive phase estimations as predictor or regularizer, the system looks for smoother phase signal estimation.

We may continue defining higher-order DPLL by adding more memory to the DPLL system. Because of their recursiveness, these DPLLs need a settling time during which the demodulated data are not valid. That is why when recursive DPLLs are used to demodulate fringe patterns the data are scanned twice in the forward and backward directions keeping the backward estimated phase as valid [1,2]. These DPLL schemes are very efficient in terms of phase estimating time due to their minimum computation overhead. This minimum computation load enables these DPLL systems to demodulate fringe patterns as the fringe data are available at video rates [1] using relatively simple dedicated digital processors. Unfortunately these systems are less robust with reference to noise because of the few data samples used to estimate the modulating phase.

\section{Non-Recursive Digital Phase Locked Loop (NRDPLL)}

Many of the above mentioned drawbacks about the recursive DPLL are solved using the proposed NDRPLL. To derive the equation of motion of Non-Recursive DPLL (NRDPLL) it is convenient to propose the system as a cost function being minimized. The proposing of a cost function is more appealing because one may add other desirable terms as we will do in the next paragraph to increase the robustness of the system. The simplest non-recursive cost function being optimized by the estimated phase $\phi_{\mathrm{e}}(x, y)$ at each site $(x, y)$ is

$U(x, y)=\sum_{(\varepsilon, \eta) \varepsilon\left(N_{x, y} \cap P\right)}\left\{I^{\prime}(\varepsilon, \eta) \cos \left[\omega_{0} \varepsilon+\phi_{\mathrm{e}}(x, y)\right]\right\}^{2}$,

$P$ is the region inside the interferogram pupil and is a two-dimensional lattice having valid fringe data inside it; $N_{x, y}$ is a neighborhood region around the coordinate $(x, y)$ where the phase is being estimated.

The actual equation of motion for the estimated phase may be found for example by fixed step gradient descent of $U(x, y)$,

$$
\begin{aligned}
\phi_{\mathrm{e}}^{k+1}(x, y)= & \phi_{\mathrm{e}}^{k}(x, y)-\tau \frac{\partial U(x, y)}{\partial \phi_{\mathrm{e}}(x, y)}, \\
\phi_{\mathrm{e}}^{k+1}(x, y)= & \phi_{\mathrm{e}}^{k}(x, y)-\tau \sum_{(\varepsilon, \eta) \varepsilon\left(N_{x, y} \cap P\right)} 2 I^{\prime}(\varepsilon, \eta) \\
& \times \sin \left[\omega_{0} \varepsilon+\phi_{\mathrm{e}}^{k}(x, y)\right] .
\end{aligned}
$$

The proposed demodulating strategy using Eq. (10) is as follows. First, the initial condition for the very first pixel $\left(x_{0}, y_{0}\right)$ which is demodulated is zero, that is,

$\phi_{\mathrm{e}}^{k=0}\left(x_{0}, y_{0}\right)=0$

with this initial condition equation (10) is iterated and after several passes (10 for example) a stable point is reached. After this, we may move to a neighborhood pixel (let us say $\left(x_{0}, y_{0}+1\right)$ ) to estimate its phase taking as initial condition the solution found at $\left(x_{0}, y_{0}\right)$, that is,

$\phi_{\mathrm{e}}^{k=0}\left(x_{0}, y_{0}+1\right)=\phi_{\mathrm{e}}^{k=10}\left(x_{0}, y_{0}\right)$. 
In other words, the last iteration at $\left(x_{0}, y_{0}\right)$ is taken as the initial condition of the following neighborhood pixel. In this way the phase estimation process is accelerated given that the last iteration of one pixel is very close to the estimated value of its neighborhood one. After the very first phase estimation it takes normally about two additional iterations to reach the stable point of Eq. (10).

Now it is useful to make a comparison with the first-order recursive DPLL. In the NDRPLL in Eq. (10) one uses not only one data term $I^{\prime}(x, y)$ instead one uses a whole two-dimensional neighborhood $N_{x, y}$ of data $I^{\prime}(\varepsilon, \eta)$ around the phase being estimated $\phi_{\mathrm{e}}(x, y)$. Also this NRDPLL has no transient response in the sense that some estimated data may not be valid. Once Eq. (10) converges it is finished, one obtains the estimation of the modulating phase and then one may move towards the next neighborhood pixel.

\section{Analysis of the NRDPLL}

To analyze this NRDPLL we will use the normalized irradiance model for $I^{\prime}(x, y)$ (Eq. (3)). Then one obtains

$$
\begin{aligned}
& \phi_{\mathrm{e}}^{k+1}(x, y) \\
& =\phi_{\mathrm{e}}^{k}(x, y)-2 \tau \sum_{(\varepsilon, \eta) \varepsilon\left(N_{x, y} \cap P\right)} \cos \left[\omega_{0} \varepsilon+\phi(\varepsilon, \eta)\right] \\
& \quad \times \sin \left[\omega_{0} \varepsilon+\phi_{\mathrm{e}}^{k}(x, y)\right],
\end{aligned}
$$

and expanding the second term we find

$$
\begin{aligned}
\phi_{\mathrm{e}}^{k+1}(x, y) & \\
= & \phi_{\mathrm{e}}^{k}(x, y)-\tau \sum_{(\varepsilon, \eta) \varepsilon\left(N_{x, y} \cap P\right)}\left\{\sin \left[\phi_{\mathrm{e}}^{k}(x, y)-\phi(\varepsilon, \eta)\right]\right. \\
& \left.+\sin \left[2 \omega_{0} \varepsilon+\phi(\varepsilon, \eta)+\phi_{\mathrm{e}}^{k}(x, y)\right]\right\} .
\end{aligned}
$$

The first term in the sum is a low frequency signal so averaging around the point $(x, y)$ will hardly affect it. On the other hand the second term is a high frequency signal having a carrier frequency twice as high as the original fringe pattern. This high frequency term is strongly attenuated by the averaging about the neighborhood $N_{x, y}$. It may be seen that the bandwidth of the NRDPLL depends on the size of the neighborhood $N_{x, y}$; the greater it is, the better rejection to the second harmonics and noise is obtained. So if the neighborhood $N_{x, y}$ is large enough $(3 \times 3$ pixels or more) then the equation of motion for the NRDPLL may be rewritten as

$$
\begin{aligned}
\phi_{\mathrm{e}}^{k+1}(x, y)= & \phi_{\mathrm{e}}^{k}(x, y)-\tau \sum_{(\varepsilon, \eta) \varepsilon\left(N_{x, y} \cap P\right)} \sin \left[\phi_{\mathrm{e}}^{k}(x, y)\right. \\
& -\phi(\varepsilon, \eta)],
\end{aligned}
$$

when the NRDPLL is working in lock the values of the estimated phase $\phi_{\mathrm{e}}(x, y)$ should be close to the values given by the true phase $\phi(\varepsilon, \eta)$ in the neighborhood $N_{x, y}$. After several iterations, the stable point on the estimated phase is reached and the estimated phase value will be close to its neighborhoods, so $\sin (x)$ may be substituted by its argument $x$ as

$\phi_{\mathrm{e}}^{\infty}(x, y)=\phi_{\mathrm{e}}^{\infty}(x, y)-\tau \sum_{(\varepsilon, \eta) \varepsilon\left(N_{x, y} \cap P\right)}\left[\phi_{\mathrm{e}}^{\infty}(x, y)-\phi(\varepsilon, \eta)\right]$,

therefore,

$\phi_{\mathrm{e}}^{\infty}(x, y)=\frac{1}{\text { Size of }\left(N_{x, y}\right)} \sum_{(\varepsilon, \eta) \varepsilon N_{x, y}} \phi(\varepsilon, \eta)$,

where the function Size of $\left(N_{x, y}\right)$ stands for the number of pixels that fall within $N_{x, y}$. For clarity purposes we have dropped out the pupil $P$ of the fringe data. As can be seen the estimated phase is equal to the average of the surrounding phase data. As a consequence the modulating phase is low pass filtered by a moving average filter acting in the phase space.

In the recursive DPLL the filtering of the high frequency term is achieved by a recursive digital filter and in the case of the NRDPLL this low pass filtering is performed by a non-recursive spatial moving average window.

\section{Regularizing the NRDPLL}

The cost function (Eq. (9)), from which the NRDPLL system was derived, measures the distance between the phase being estimated $\phi_{\mathrm{e}}(x, y)$ and its neighborhood data $\phi(\varepsilon, \eta)$ in the wrapped space. So "near" phase values in the wrapped space may have still a $2 n \pi$ radians $(n=1,2, \ldots)$ of 
phase difference in the Euclidean space. However this situation is attenuated by the fact of propagating the stable estimated phase value as the initial condition of the next phase pixel to be optimized. Unfortunately computer simulations using noisy fringe patterns easily demonstrate that some demodulated phase values do have a $2 n \pi$ error among neighborhood pixels. If by chance this occurs, the cost function as stated in Eq. (9) will not see them because the measure of the neighborhood distance is wrapped, so no driving force is reported to the equation of motion of the NRDPLL system to correct these spurious jumps.

A useful way of solving these spurious $2 n \pi$ jumps in the Euclidean space is by regularizing the cost function where the NRDPLL is derived (Eq. (9)). This is usually made by weighting the Euclidean distance between the currently estimated phase and its neighborhood demodulated phases. So adding to this energy function $U(x, y)$ such a regularizing term, the final cost function will have the following form:

$$
\begin{aligned}
U(x, y)= & \sum_{(\varepsilon, \eta) \varepsilon\left(N_{x, y} \cap P\right)}\left\{I^{\prime}(\varepsilon, \eta) \cos \left[\omega_{0} \varepsilon+\phi_{\mathrm{e}}^{k}(x, y)\right]\right\}^{2} \\
& +\sum_{(\varepsilon, \eta) \varepsilon\left(N_{x, y} \cap P\right)}\left[\phi_{\mathrm{e}}(\varepsilon, \eta)-\phi_{\mathrm{e}}(x, y)\right]^{2} m(\varepsilon, \eta),
\end{aligned}
$$

where $m(x, y)$ is an indicator field which equals 1 if the site $(x, y)$ has already been phase estimated, and zero otherwise. As can be seen this new cost function also measures the Euclidean distance between the currently estimated phase and its previously estimated ones. The equation of motion for this regularized NRDPLL is obtained again using fixed step gradient descent to give

$$
\begin{aligned}
& \phi_{\mathrm{e}}^{k+1}(x, y) \\
& =\phi_{\mathrm{e}}^{k}(x, y) \\
& \quad-\tau \sum_{(\varepsilon, \eta) \varepsilon\left(N_{x, y} \cap P\right)}\left\{2 I^{\prime}(\varepsilon, \eta) \sin \left[\omega_{0} \varepsilon+\phi_{\mathrm{e}}^{k}(x, y)\right]\right. \\
& \left.\quad+2\left[\phi_{\mathrm{e}}(\varepsilon, \eta)-\phi_{\mathrm{e}}^{k}(x, y)\right] m(\varepsilon, \eta)\right\} .
\end{aligned}
$$

Using the normalized fringe pattern in Eq. (3), expanding the multiplicative term, and dropping down the high frequency term attenuated by the averaging about $N_{x, y}$ the equation of motion looks as

$$
\begin{aligned}
\phi_{\mathrm{e}}^{k+1}(x, y) & \\
= & \phi_{\mathrm{e}}^{k}(x, y) \\
& -\tau \sum_{(\varepsilon, \eta) \varepsilon\left(N_{x, y} \cap P\right)}\left\{\sin \left[\phi_{\mathrm{e}}^{k}(x, y)-\phi(\varepsilon, \eta)\right]\right. \\
& \left.+2\left[\phi_{\mathrm{e}}(\varepsilon, \eta)-\phi_{\mathrm{e}}^{k}(x, y)\right] m(\varepsilon, \eta)\right\} .
\end{aligned}
$$

Then the estimated phase will move towards decreasing the wrapped distance between the phase being estimated and its neighborhood through the sine function, and a term (the regularizer) that decreases the Euclidean distance between the estimated phase and the previously estimated neighborhood. In other words, the above equation of motion has a driving force proportional to the Euclidean distance between the current estimated phase and its neighborhood discouraging any tendency to create spurious $2 n \pi$ phase jumps. When discussing the first-order recursive DPLL we saw that it also has a linear regularizing (or linear predictor) term given by the previously estimated phase. However, this simple regularizing term enforces the condition of estimating a phase having a similar value to the previously demodulated phase. When the neighborhood $N_{x, y}$ is large (more than $5 \times 5$ pixels) the estimated phase tends to be flattened distorting the estimated solution. A solution to this problem is presented in the next section.

\section{Non-recursive phase and frequency locked loop}

In this section we will continue with the improvement of the proposed NRDPLL by adding some new elements to the more elementary NRDPLL that was presented in the last section. The NRDPLL just presented cannot cope with high amounts of noise because the neighborhood $N_{x, y}$ cannot be made large enough to obtain a very narrow bandwidth. This is because the use of just the phase value as linear predictor is not good enough. When the size of $N_{x, y}$ is large using as regularizer (or predictor) only neighborhood phase values, the demodulated phase tends to be flat. In 
this section we will instead fit a tilted plane to the demodulated phase and use this plane as predictor. Therefore the extended NRDPLL model will need to estimate not only the local phase but also the local frequencies along the $x$ and $y$ directions which are the slopes of the tilted plane used as predictor.

In this extended NRDPLL model one assumes that locally the intensity of the fringe pattern may be considered as spatially mono-chromatic, so its irradiance may be modeled as a sinusoidal function phase-modulated by a plane $p(\cdot)$ (the fitting plane). Specifically, the proposed cost function to be minimized by the estimated phase $\phi_{\mathrm{e}}(x, y)$ and frequencies $\omega_{x}(x, y)$ and $\omega_{y}(x, y)$ at each site $(x, y)$ is

$$
\begin{aligned}
U(x, y)= & U_{1}(x, y)+U_{2}(x, y) \\
= & \sum_{(\varepsilon, \eta) \varepsilon\left(N_{x, y} \cap P\right)}\left\{I ^ { \prime } ( \varepsilon , \eta ) \operatorname { c o s } \left[\omega_{0} \varepsilon\right.\right. \\
& +p(x, y, \varepsilon, \eta)]\}^{2}+\sum_{(\varepsilon, \eta) \varepsilon\left(N_{x, y} \cap P\right)}\left[\phi_{\mathrm{e}}(\varepsilon, \eta)\right. \\
& -p(x, y, \varepsilon, \eta)]^{2} m(\varepsilon, \eta),
\end{aligned}
$$

where

$$
\begin{aligned}
p(x, y, \varepsilon, \eta)= & \phi_{\mathrm{e}}(x, y)+\omega_{x}(x, y)(x-\varepsilon) \\
& +\omega_{y}(x, y)(y-\eta),
\end{aligned}
$$

some of the variables appearing above have already been defined, so we will define those not yet mentioned. The functions $\omega_{x}(x, y)$ and $\omega_{y}(x, y)$ are the estimated local frequencies (slopes of $\phi(x, y)$ ) along the $x$ and $y$ directions, respectively. As in the other NRDPLL this cost function is made of two parts; the first part is associated with the fringe data $I^{\prime}(\varepsilon, \eta)$ (Eq. (3)) and the second one is the regularizer (or predictor plane) which uses previously estimated phase pixels $\phi_{\mathrm{e}}(\varepsilon, \eta)$.

As we did before, the equation of motion of this NRDPLL may be found by optimizing $U(x, y)$ (Eqs. (21) and (22)). To demodulate a given carrier frequency fringe pattern we need to find the minimum of the cost function $U(x, y)$ at each site $(x, y)$ with respect to the variables $\phi_{0}(x, y), \omega_{x}(x, y)$ and $\omega_{y}(x, y)$. Unfortunately the cost function $U(x, y)$ in Eq. (21) is non-convex so fast optimizing techniques are precluded. Therefore we normally use fixed-step gradient descent to find the optimum values for the searched variables. Therefore, given $\mathbf{r}(x, y)=\left(\phi_{0}, \omega_{x}, \omega_{y}\right)^{\mathrm{T}}$ we then estimate the phase and frequency at each site $(x, y)$ according to

$\mathbf{r}^{k+1}(x, y)=\mathbf{r}^{k}(x, y)-\tau \nabla_{r} U(x, y)$,

where $\tau$ is the constant step of descent of the gradient search. The symbol $\nabla_{r}$ is the gradient of $U(x, y)$ with reference to the three components of $\mathbf{r}(x, y)=\left(\phi_{\mathrm{e}}, \omega_{x}, \omega_{y}\right)^{\mathrm{T}}$, that is,

$\nabla_{r} U(x, y)=\left(\frac{\partial U(x, y)}{\partial \phi_{\mathrm{e}}(x, y)}, \frac{\partial U(x, y)}{\partial \omega_{x}(x, y)}, \frac{\partial U(x, y)}{\partial \omega_{y}(x, y)}\right)$.

The constant phase step $\tau$ for the $\phi_{\mathrm{e}}(x, y)$ component in Eq. (23) may be multiplied by 10 to accelerate the convergence rate. The gradient search method also needs a starting condition for $\mathbf{r}^{0}$ in Eq. (23). To demodulate the initial pixel (seed pixel) at $\left(x_{0}, y_{0}\right)$ inside $P$ we have chosen as initial condition the vector $\mathbf{r}^{0}\left(x_{0}, y_{0}\right)=(0,0,0)^{\mathrm{T}}$. When the seed vector at $\mathbf{r}^{0}\left(x_{0}, y_{0}\right)$ is optimized, the regularizing term in $U(x, y)$ is not taken into account given that the seed pixel is the first one that is demodulated and, as a consequence the indicator function $m(x, y)$ equals zero all over the pupil $P$. Once the seed vector $\mathbf{r}\left(x_{0}, y_{0}\right)$ is estimated (using Eqs. (21) and (23)) we may proceed sequentially with the rest of the sites in $P$. Then NRDPLL system must follow a continuous sequential path starting at the seed position; propagating the final condition of $\mathbf{r}\left(x_{0}, y_{0}\right)$ in Eq. (23) as the initial condition for the following adjacent site. As mentioned, the fact of using the stable condition of a given site as initial condition for the adjacent one has two immediate advantages. The first one is a fast convergence rate given that the solution is near the desired value. The second one is that the unwrapping process is achieved implicitly. Using this scanning strategy the NRDPLL proceeds from pixel to pixel until the whole interferogram is phase demodulated.

We should remark several things about the applicability and limitations of the NRDPLL that have not been mentioned so far, these are: 
- The implicit unwrapping within the NRDPLL is quite robust and it is comparable to the least-squares based algorithm $[7,8]$.

- The robustness of the NRDPLL is proportional to the size of $N_{x, y}$ but a large neighborhood $N_{x, y}$ may reduce the true dynamic range of the estimated phase by low pass overfiltering.

- The phase singularities that the NRDPLL can properly handle are those generated by random phase noise. Other singularities such as abrupt phase jumps are not properly demodulated.

- By properly choosing the convergence parameter the stability is not compromised by enlarging the neighborhood $N_{x, y}$ to a reasonably large value. What it may happen is a reduction in the dynamic range of the estimated phase because the signal is overfiltered. Also the optimizing of a large neighborhood will take more time which may be unacceptably high.

\section{Analyzing the non-recursive phase and frequency locked loop}

To analyze the recurrence equations given by Eq. (23) we need the partial derivatives with respect to $\phi_{\mathrm{e}}(x, y), \omega_{x}(x, y)$ and $\omega_{y}(x, y)$, and also the normalized irradiance (Eq. (3)) for the fringe model. As in the other cases, the first term of the fringe-reference product is a low frequency term so the averaging around the point $(x, y)$ will not affect it. On the other hand the second term is a high frequency term having a carrier frequency twice as much as the original fringe pattern. This high frequency term is strongly attenuated by the averaging about the neighborhood $N_{x, y}$. Then, after filtering out the high frequency term, we obtain the recurrence equation for the phase $\phi_{\mathrm{e}}(x, y)$ as

$$
\begin{aligned}
\phi_{\mathrm{e}}^{k+1}(x, y) & \\
= & \phi_{\mathrm{e}}^{k}(x, y) \\
& +\tau \sum_{(\varepsilon, \eta) \varepsilon\left(N_{x, y} \cap P\right)}\left\{\sin \left[\phi(\varepsilon, \eta)-p^{k}(x, y, \varepsilon, \eta)\right]\right. \\
& \left.+2\left[\phi_{\mathrm{e}}^{k}(\varepsilon, \eta)-p^{k}(x, y, \varepsilon, \eta)\right] m(\varepsilon, \eta)\right\},
\end{aligned}
$$

where $p^{k}(x, y, \varepsilon, \eta)$ is the plane defined in Eq. (22) at the iteration $k$. As can be seen from this equa- tion the plane $p^{k}(\cdot)$ will follow (fit) the values of the phase data $\phi(\varepsilon, \eta)$ around the site $(x, y)$ within the neighborhood $N_{x, y}$. Continuing with the local frequencies the recurrence equation for $\omega_{x}(x, y)$ is

$$
\begin{aligned}
& \omega_{x}^{k+1}(x, y) \\
& =\omega_{x}^{k}(x, y) \\
& \quad+\tau \sum_{(\varepsilon, \eta) \varepsilon\left(N_{x, y} \cap P\right)}\left\{\sin \left[\phi(\varepsilon, \eta)-p^{k}(x, y, \varepsilon, \eta)\right](x-\varepsilon)\right. \\
& \left.\quad+2\left[\phi_{\mathrm{e}}^{k}(\varepsilon, \eta)-p^{k}(x, y, \varepsilon, \eta)\right](x-\varepsilon) m(\varepsilon, \eta)\right\} .
\end{aligned}
$$

And finally the recurrence equation for $\omega_{y}(x, y)$ is

$$
\begin{aligned}
& \omega_{y}^{k+1}(x, y) \\
& =\omega_{y}^{k}(x, y) \\
& \quad+\tau \sum_{(\varepsilon, \eta) \varepsilon\left(N_{x, y} \cap P\right)}\left\{\sin \left[\phi(\varepsilon, \eta)-p^{k}(x, y, \varepsilon, \eta)\right](y-\eta)\right. \\
& \left.\quad+2\left[\phi_{\mathrm{e}}^{k}(\varepsilon, \eta)-p^{k}(x, y, \varepsilon, \eta)\right](y-\varepsilon) m(\varepsilon, \eta)\right\} .
\end{aligned}
$$

In all cases the driving force of the NRDPLL is the difference between the plane $p(x, y, \varepsilon, \eta)$ and the values of the phase $\phi(\varepsilon, \eta)$ around $(x, y)$. Whenever a difference exists between $\phi(\varepsilon, \eta)$ and the fitted plane $p(x, y, \varepsilon, \eta)$ the system will continue to evolve. In other words when the NRDPLL is working in lock the plane $p(x, y, \varepsilon, \eta)$ will closely follow the values $\phi(\varepsilon, \eta)$ around $(x, y)$.

So as before, the bandwidth of this NRDPLL depends on the size of the neighborhood $N_{x, y}$; the greater it is the better rejection to the second harmonics and noise is obtained. However $N_{x, y}$ cannot be too large otherwise an over-filtering of the demodulated signal is obtained. The regularizing term is used to improve the stability of the NRDPLL system discouraging $2 n \pi$ spurious phase jumps.

\section{Examples of application of the NRDPLL}

We start by analyzing some computer simulation results. In Fig. 2(a) we have a computer generated noisy fringe pattern. In Fig. 2(b) we show the resulting estimated phase when the Fourier [3] technique is used and in Fig. 2(c) shows the estimated phase given by the non-recursive 
phase and frequency locked loop (Eq. (23)). The phase given by the NRDPLL was wrapped again for comparison purposes with the wrapped phase obtained by the Fourier technique. For this Fourier filtering we tried several bandwidths and we kept the best visual result. From Fig. 2 it is clear that the noise rejection capacity of the NRDPLL is far superior to the one obtained using the Fourier technique. We used as a neighborhood $N_{x, y}$ equal to 17 pixels wide in the $x$ direction and 3 pixels high in the $y$ direction. The two-dimensional scanning of the fringe pattern was achieved in a row by row basis until the whole fringe pattern was demodulated.

The better results obtained by the use of the herein presented phase locked loop may also be seen from Fig. 3. In this figure we represent a wide band fringe spectrum being filtered by a pass-band quadrature Fourier filter and also by a narrow loop filter within the phase tracking system (NRDPLL). While the Fourier filter must have the

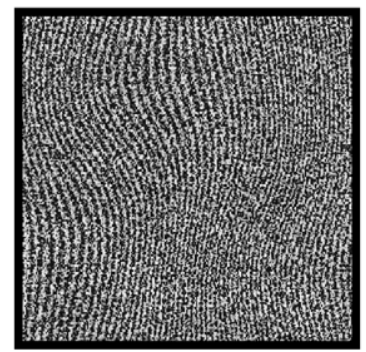

(a)
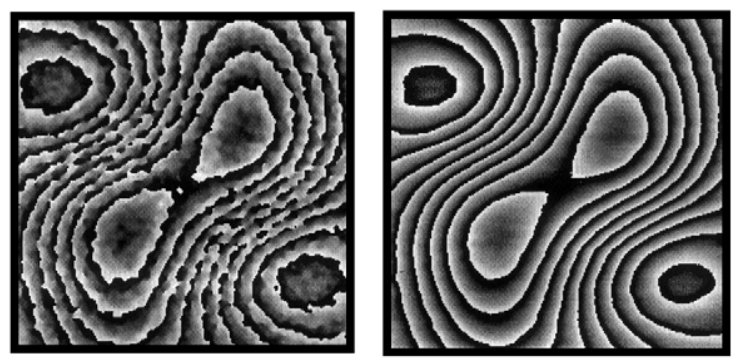

(b)

(c)

Fig. 2. Computer generated experiment: (a) Input noisy fringe pattern. (b) Demodulated fringe pattern according to the Fourier technique using the "best" quadrature filter in order to reject as much noise as possible while preserving the data form. (c) Demodulated fringe pattern using the proposed NRDPLL a neighborhood $N_{x, y}$ of $7 \times 3$ pixels was used. The obtained phase was re-wrapped to compare it against the Fourier result.

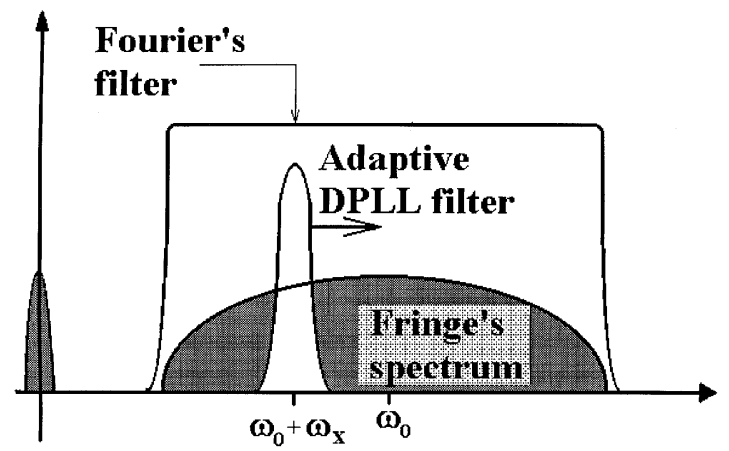

Fig. 3. Schematic view of the spectrum of wide-band carrier fringes. The Fourier quadrature filter must be as wide as the whole signal spectrum, while the DPLL may have a band pass narrower than the signal spectrum.

same bandwidth as the fringes spectrum, the phase tracking system must have an adaptive narrow bandwidth filter which tracks the local phase and frequency of the signal.

In Fig. 4(a) we show a fringe pattern projected over a dummy face. In Fig. 4(b) we show the information on the shadows on the projected light. This is made in order to demodulate first the whiter zones (which have a better signal to noise ratio, [6]) and afterwards the fringes falling at the shadow regions where the projected fringes have low modulation. Using this two-dimensional scanning strategy [6] one demodulates the good fringes first and after this the fringes with a lower signal to noise ratio are processed. In this way the bad fringes (those at the shadows) cannot corrupt the areas where good fringes are easily demodulated (the whiter zones). In this case we have defined three quality or signal to noise levels; the white one (highest quality), the gray one (medium quality) and the dark one (lowest quality). Using these qualities one demodulates in the first place the highest quality fringes and in the last place the darkest zones which contain the lowest quality ones [6]. The result of applying the proposed NRDPLL is given in Fig. 5. In this case we have used a neighborhood $N_{x, y}$ of 13 pixels wide in the $x$ direction and 3 pixels wide in the $y$ direction.

In Fig. 6 we show the result of demodulating the fringe pattern using the Synchronous technique [4] and using the least-squares unwrapping 


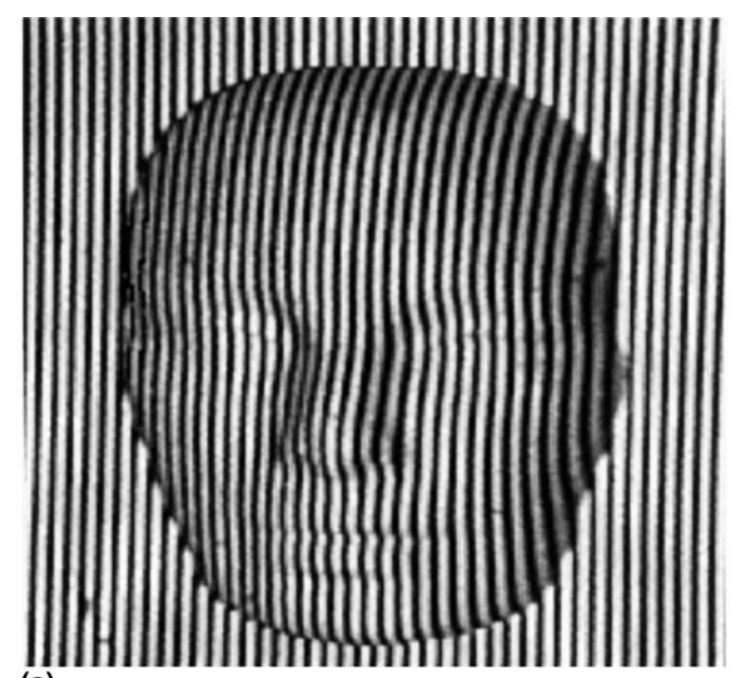

(a)

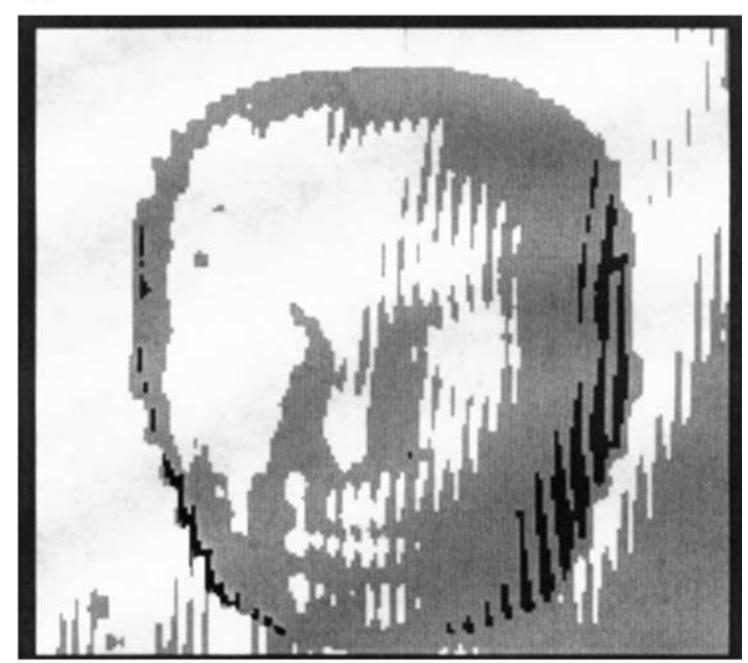

(b)

Fig. 4. (a) Experimentally obtained projection fringes over a dummy face. (b) Quality (contrast) of the fringes according to three quality levels.

technique [7] to obtain the continuous demodulated phase. The demodulated face shown in this figure is not as good as the one obtained by the NRDPLL shown in Fig. 5.

In Fig. 7 we show the result of demodulating the fringe pattern using the Fourier technique [3] and to unwrap the demodulated phase the leastsquares unwrapping method [7] was used. Also, the demodulated quality shown is lower than the one obtained by the NRDPLL shown in Fig. 5

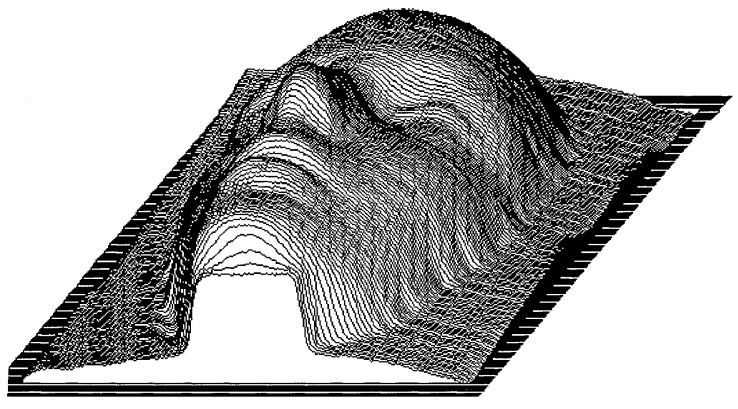

Fig. 5. Demodulated signal using the herein proposed non-recursive digital phase locked loop.

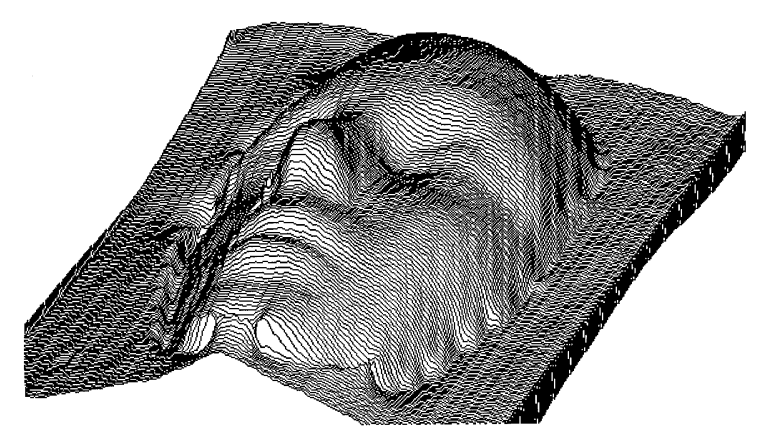

Fig. 6. Demodulated signal using the Synchronous method and the least-squares unwrapping technique.

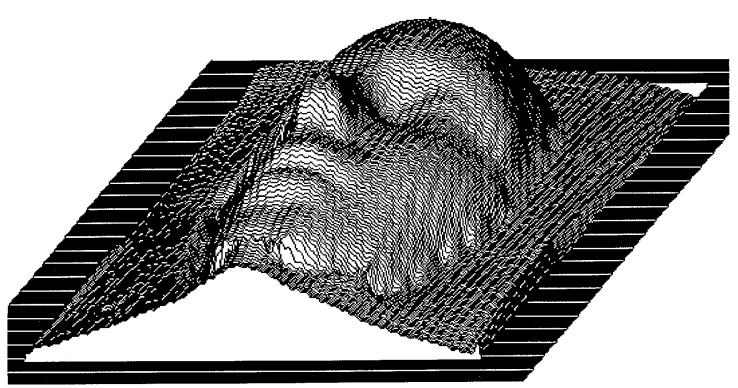

Fig. 7. Demodulated signal using the Fourier method and the least-squares unwrapping technique.

as can be seen for example from the face boundaries.

The computing times required to demodulate the fringe patterns presented in these experiments using a $500 \mathrm{MHz}$ pentium PC were: $6 \mathrm{~s}$ for the Fourier technique, also about $6 \mathrm{~s}$ for the Synchronous method and finally almost $7 \mathrm{~s}$ for the NRDPLL. 


\section{Conclusion}

We have presented a new non-recursive twodimensional digital phase locked loop (NRDPLL). The main advantage of the presented approach with respect to previous recursive phase locked loop is its far superior performance on noise rejection and stability in the estimated phase. Also the NRDPLL has very good phase estimation properties at the edge of the interferogram's pupil which is much better than the Fourier and the Synchronous techniques.

However, the main drawbacks of the NRDPLL are: (a) the carrier frequency must be high enough (greater than $\pi / 2$ radians per pixel) to properly reject the second harmonic signal generated by the product between the interferogram and the reference signal; (b) the NRDPLL system cannot deal with abrupt spatial phase changes or jumps. This is because the running average estimation of the modulating phase will smooth out the phase jump over several other phases and finally; (c) if the twodimensional neighborhood $N_{x, y}$ is too large the demodulated phase may be overfiltered and the phase signal will have a diminished dynamic range.

The NRDPLL was also compared against wellestablished techniques such as the Fourier method and the Synchronous technique. It was shown that the proposed NRDPLL outperforms these traditional approaches. The reason for this to happen resides in the fact that the NRDPLL may be seen as an adaptive narrow band-pass filter which tracks the local phase and frequency of a signal having a wider spectrum. This narrow adaptive band-pass filter translates into a high gain in the signal to noise ratio of the detected phase.

\section{Acknowledgements}

We would like to acknowledge the Mexican government support through CONACYT for the development of this work.

\section{References}

[1] M.A. Gdeisat, D.R. Burton, M.J. Lalor, Appl. Opt. 39 (2000) 5326.

[2] M. Servin, R. Rodriguez-Vera, J. Mod. Opt. 40 (1993) 2087.

[3] M. Takeda, H. Ina, S. Kobayashi, J. Opt. Soc. Am. 72 (1982) 156.

[4] K.H. Womack, Opt. Eng. 23 (1984) 391.

[5] J. Kozlowwski, O. Serra, Opt. Eng. 36 (1996) 2025.

[6] J. Villa, J.A. Quiroga, M. Servin, Appl. Opt. 39 (2000) 502.

[7] D.C. Ghiglia, L.A. Romero, Opt. Soc. Am. A 11 (1994) 107.

[8] M. Servin, F.J. Cuevas, D. Malacara, J.L. Marroquin, Appl. Opt. 38 (1999) 1934. 